Elke Heizmann, Albrecht Wacker, Gabriele Strobel-Eisele, Melanie Döring, Jochen Kramer \& Christian Rietz

\title{
Der Einfluss von Kooperation, Interesse und Motivation auf die Leistungserbringung in kooperativen Schulabschlussprüfungen
}

\section{Zusammenfassung}

Kooperative Prüfungsformate sind Bestandteile bei Schulabschlussprüfungen. Bislang ungeklärt ist, ob die kooperative Anlage in einem Zusammenhang mit der erzielten Note steht. Die Ergebnisse einer durchgeführten Schülerbefragung (n=1.670) im Rahmen einer kooperativen Abschlussprüfung zur Erreichung der Mittleren Reife deuten darauf hin, dass für die Leistungsunterschiede in der Prüfungsnote die Kooperation der Lernenden sowie ihr Interesse bedeutsam sind.

Schlüsselwörter: Kooperation, Interesse, Motivation, Abschlussprüfung, Sekundarstufe I

\section{The Influence of Co-operation, Interest and Motivation on Performance in Co-operative School-Leaving Examinations}

\begin{abstract}
Co-operative examination formats are contents at school-leaving examinations. So far it is unexplained if the co-operative system stays in correlation with the achieved mark. The results of a conducted student survey $(n=1,670)$, within the scope of a co-operative school-leaving examination to achieve the graduation "Mittlere Reife" [intermediate school-leaving certificate], indicates that co-operation and interest are significant for performance differences of the examination mark.

Keywords: co-operation, interest, motivation, school-leaving examinations, lower secondary education
\end{abstract}




\section{Einleitung}

Kooperative Kompetenzen gelten als Schlüsselerfolgsfaktoren, um hochkomplexe Problemstellungen der Gegenwart und Zukunft bewältigen zu können (Müller \& Bungard, 2013). Relevant sind sie vor allem in der Teamarbeit, die sich in allen Bereichen der Wissensgesellschaft durchgesetzt hat. Aber auch für ein friedliches Zusammenleben in einer zunehmend kulturell heterogenen Umgebung sind sie unabdingbar (Kanning, 2009). Im schulischen Erziehungs- und Bildungsauftrag wird ihnen deshalb ein hoher Stellenwert zugesprochen, der sich in ihrer Zertifizierung in schulischen Abschlussprüfungen manifestiert.

Studien zeigen, dass ein nach den Prinzipien des Kooperativen Lernens gestalteter Gruppenunterricht einen positiven Einfluss auf die Ausbildung kooperativer Fähigkeiten ausüben kann (Johnson \& Johnson, 2006). Kooperatives Lernen (KL) umfasst Unterrichtskonzepte, für die nicht nur hohe Lernfortschritte belegt sind, sondern auch positive Effekte auf kooperative Kompetenzen mehrfach herausgearbeitet werden konnten und zudem ein primärer Effekt auf die Verstärkung von Interesse, Motivation und Problemlösefähigkeit nachgewiesen wurde (vgl. Hattie, 2013).

In den letzten Jahren ist ein vermehrter Einsatz von KL in der Unterrichtspraxis zu verzeichnen (vgl. Völlinger, Supanc \& Brunstein, 2018) und in dessen Folge eine zunehmende Implementation kooperativer Arrangements in Schulabschlussprüfungen. Mittlerweile sind in acht Ländern der Bundesrepublik Gruppenprüfungen eingeführt. ${ }^{1}$ Ihr Ziel ist es, neben den fachlichen Leistungen der Prüflinge die während der Schulzeit geförderten überfachlichen Fähigkeiten - wie die Fähigkeit, in einem Team komplexe Aufgaben zu bewältigen - im Abschlusszeugnis zu zertifizieren.

Für die kooperativen Assessments ist die Frage ungeklärt, in welchem Zusammenhang die Kooperation mit der Note steht und ob das Interesse und die Motivation die Note beeinflussen. Hier setzt der vorliegende Beitrag am Beispiel der sogenannten „Fächerübergreifenden Kompetenzprüfung“ an - einer für alle Realschulprüflinge verpflichtenden kooperativen Gruppenprüfung zur Erreichung der Mittleren Reife.

Der Artikel ist wie folgt aufgebaut: Einleitend werden die theoretischen Grundlagen dargelegt und kooperative Lernsettings und ihre Modellierung im Prüfungsprozess beschrieben (1). Anschließend werden die Forschungsfragen und das methodische Vorgehen berichtet (2 und 3). Der Darlegung der Ergebnisse (4) folgt im letzten Abschnitt eine Diskussion (5).

1 Es handelt sich um: Baden-Württemberg, Bayern, Berlin, Bremen, Mecklenburg-Vorpommern, das Saarland, Schleswig-Holstein und Thüringen. 
Der Einfluss von Kooperation, Interesse und Motivation auf die Leistungserbringung |

\section{Kooperative Prüfungen: theoretische Bezüge}

\subsection{Kooperative Lernsettings und ihre Modellierung in Prüfungsformaten}

Die Fähigkeit zu kooperieren wird in der Literatur als ein Teilbereich sozialer Kompetenz diskutiert, welche sich aus verschiedenen kognitiven, emotionalen und motivationalen Aspekten zusammensetzt und deren spezifische Konstruktkriterien als ungeklärt gelten. Nach Kunter, Stanat und Klieme (2005) lassen sich in schulischen Gruppensettings solche kooperativen Kompetenzen als erfolgreich bewerten, in denen es Lernenden gelingt, positive soziale Beziehungen zu gestalten, ohne dabei eigene Zielsetzungen zu vernachlässigen.

Dieser Grundgedanke spiegelt sich auch als zentrales Merkmal kooperativer Arbeitsformen wider, einer Interaktionsform, in der kleine Gruppen gemeinsam und im wechselseitigen Austausch lernen und auf ein von allen geteiltes Ziel hinarbeiten (Konrad \& Traub, 2010). Der soziale Prozess ist bei dieser Form des Lernens der wichtigste Bestandteil. Nach soziokonstruktivistischen Ansätzen (Vygotsky, 1978) wird Lernen als Folge kognitiver Konflikte erklärt, welche insbesondere durch das wechselseitige Erklären ausgelöst werden können (Slavin, 1993).

Mit dem Einsatz kooperativer Arbeitsformen wird eine Initiierung dieser lernrelevanten Prozesse angestrebt. Vor allem aber soll diese Arbeitsform den Lernenden die Möglichkeit bieten, ihre kooperativen Fähigkeiten weiterzuentwickeln, indem die Vermittlung von Fachwissen mit der Gestaltung sozialer Beziehungen verbunden wird (Dubs, 2009). In kooperativen Abschlussprüfungen stehen die Kooperationskompetenzen der Prüflinge im Vordergrund (z.B. MKJS BW, 2006, S. 27). Es geht um die Fähigkeit, die Lösung zu einer komplexen Themenstellung in Kooperation mit anderen Personen zu erarbeiten und darzustellen.

In Baden-Württemberg absolvieren Realschulabgänger ${ }^{\star}$ innen verpflichtend eine kooperative mündliche Abschlussprüfung, die „Fächerübergreifende Kompetenzprüfung“ (FüK). Hierzu bearbeiten sie in Kleingruppen die Inhalte einer selbstgewählten Fragestellung und präsentieren ihre Ergebnisse in der Gruppe. Es handelt sich dabei um eine kooperative Lernform, bei der sich die Lernenden zunächst für einen Teilbereich des Themas „Expertenwissen“ aneignen und sich dieses im intensiven Austausch wechselseitig vermitteln (vgl. Huber, 2007). Als eine der wichtigsten Voraussetzungen für eine lernwirksame Kooperation in diesen Settings gilt die positive Abhängigkeit bei gleichzeitiger individueller Verantwortlichkeit (Slavin, 1993). Durch die Aufteilung in „Expertenbereiche“ ist der individuelle Beitrag jedes Prüflings klar ersichtlich; das Setting setzt aber gleichzeitig die koordinierte Zusammenarbeit voraus, denn für das Ergebnis ist der Beitrag aller Mitglieder notwendig. 
Gefordert wird in der Prüfung die Beantwortung der Fragestellung, bei der durch die Verknüpfung aller Themenbereiche aus unterschiedlichen fachlichen Perspektiven heraus sowohl jeder Prüfling seine persönliche Expertise unter Beweis stellt als auch die Gruppe zeigt, inwieweit die Thematik vertieft, eine Meinung gebildet und Alternativen entwickelt und reflektiert wurden (vgl. MKJS BW, 2006, S. 27).

Für jegliches schulisches Lernen ist das individuelle Interesse ausschlaggebend (vgl. Schiefele, 1991). Wer aus Interesse handelt, empfindet selbst mühevolle Auseinandersetzungen mit einem Thema als befriedigend, nutzt elaboriertere Lernstrategien und investiert insgesamt mehr Energie für das Lernen. Laut Schiefele (1991) schreiben Personen ihren Interessensgegenständen eine hohe subjektive Bedeutung zu und verbinden positive Emotionen damit. Nur wenn diese Komponenten positiv erlebt werden, kann sich Interesse entwickeln (Krapp, 2005). Dies erfordert nach der Selbstbestimmungstheorie (Deci \& Ryan, 2002) die Befriedigung dreier menschlicher Grundbedürfnisse, wozu KL optimale Bedingungen liefern kann (Krapp, 2008): Lernende, die sich als kompetent (z. B. durch das Einbringen von Wissen als „Experte“ bzw. „Expertin“), selbstbestimmt und sozial eingebunden erleben, sind höher motiviert und erzielen bessere Ergebnisse. Die Beachtung dieser Bedürfnisse gilt als eine zentrale Voraussetzung für die Entstehung und Aufrechterhaltung von intrinsischer Lernmotivation und fachlichen Interessen (Krapp, 2005). Die selbstbestimmte kooperative Arbeitsweise in der FüK fördert ein interessengeleitetes Arbeiten - bspw. durch vielfältige Wahl- und Handlungsmöglichkeiten - und müsste sich demnach in besonderer Weise auf die Motivation und das Interesse auswirken und damit auch einen Einfluss auf die Note haben.

Prüfungsgegenstand der FüK sind die Gruppenpräsentation und ein darauf folgendes Prüfungsgespräch (pro Prüfling 15 Minuten). Alle Inhalte müssen aus dem Stoffgebiet zweier Fächer oder Fächerverbünde der 9. und 10. Klassenstufe stammen. Jeder Prüfling erhält am Ende eine individuelle, ganze Note. Zwei Lehrkräfte begleiten den Arbeitsprozess, der so eigenständig wie möglich verlaufen sollte.

Die Stärke dieses Prüfungsformats wird in der Kooperation gesehen, die von den Lernenden u. a. die wechselseitige Unterstützung, das Vertreten der eigenen Meinung und das Akzeptieren anderer Meinungen, die Einnahme verschiedener Perspektiven oder das Treffen eigenständiger Entscheidungen erfordert (MKJS BW, 2006, S. 27). Diese komplexen Kooperationsfähigkeiten werden in der Theorie des KL nicht als selbstverständlich vorausgesetzt; vielmehr wird deren gezielte Förderung explizit als Basiselement (Johnson \& Johnson, 2006) und als fester Bestandteil des Unterrichtsalltags gefordert (Reinmann \& Mandl, 2006).

Praxisnahe Literatur bietet didaktische Möglichkeiten, um KL einzusetzen, bzw. Trainingsmaßnahmen, um die benötigten kooperativen Fähigkeiten gezielt anzubahnen (z.B. Brüning \& Saum, 2009a; Brüning \& Saum, 2009b; Borsch, 2015; Green \& 
Der Einfluss von Kooperation, Interesse und Motivation auf die Leistungserbringung |

Green, 2005). Auch im Programm SINUS-Transfer (Steigerung der Effizienz des mathematisch-naturwissenschaftlichen Unterrichts) zur Unterrichtsentwicklung steht die Förderung lernwirksamer Kooperation aus der domänenspezifischen Perspektive im Fokus. Systematische Unterstützung für Schulen bei der Implementation einer kooperativen Lernkultur bieten Weiterbildungsprogramme wie bspw. COOL (Co-Operative Open Learning, 2018).

Ungeachtet der Fülle an Materialien ist dennoch unzureichend geklärt, welche speziellen Fähigkeiten Lernende für eine lernwirksame Kooperation benötigen (Kunter, Stanat \& Klieme, 2005) bzw. mit welchen Maßnahmen die Kooperationen am besten gefördert werden können (Reinmann \& Mandl, 2006). Die Beseitigung dieses Defizits ist für die gemeinsame Arbeit von großer Bedeutung, da die komplexen Anforderungen den Verlauf des gemeinsamen Prozesses maßgeblich mitbestimmen und anzunehmen ist, dass ihnen ein Einfluss auf die Note zukommt.

\subsection{Leistungsfaktoren kooperativer (Prüfungs-)Arrangements}

Schulleistungen werden in der Lernforschung multikausal determiniert. Mögliche Faktoren repräsentiert das Angebot-Nutzungs-Modell nach Helmke (2015; zur Erläuterung vgl. Kohler \& Wacker, 2013). Dabei wird angenommen, dass zwischen den Beteiligten eine reziproke Angebot-Nutzungs-Struktur bestehe, deren „Angebote“ nur dann zu einer Wirkung führten, wenn sie Lernende zu motivationalen sowie kognitiven Prozessen anstießen.

Für kooperative Prüfungsformen lassen sich folgende Komponenten ableiten:

1. Lernpotenzial: Das individuelle Lernpotenzial umfasst kognitive, motivationale und volitionale Schülermerkmale. Vor allem bei steigender Komplexität von Aufgaben gilt das spezifische Vorwissen als wichtigstes kognitives Lernermerkmal (Helmke, 2015). Zusammenhänge zwischen Leistung und Interesse sowie Motivation sind empirisch mehrfach nachgewiesen (z. B. Marsh, Trautwein, Lüdtke, Köller \& Baumert, 2005). Für KL konnten primäre Effekte auf die Verstärkung von Interesse und Motivation belegt werden (vgl. Hattie, 2013, S. 251).

2. Lernaktivitäten („Nutzung“): Empirisch ist ein positiver Zusammenhang zwischen der Unterrichtszeit und dem Lernzuwachs nachgewiesen (vgl. Helmke, 2015). In dieser Studie wird die Lernzeit durch die Anzahl der Gruppentreffen operationalisiert.

3. Gruppenarbeit („Angebot“): Gruppenarbeit stellt eine wichtige Lerngelegenheit dar (Helmke, 2015). Insbesondere unter kooperativen Bedingungen besitzen interpersonale Beziehungen einen bedeutenden Einfluss auf die Leistung (Hamm \& Faircloth, 2005). 
Die nachfolgende Untersuchung orientiert sich an diesem Modell und greift die benannten Merkmale in einer linearen Regressionsanalyse auf.

\subsection{Forschungsstand}

Studien zu KL zeigen positive Effekte auf die Produktivität der Lernenden, sowohl für kognitive und motivationale als auch für soziale Zielkriterien (vgl. Hattie, 2013, S. 250). Die leistungsförderliche Wirkung bedarf allerdings sowohl der Berücksichtigung der beschriebenen interdependenten Bedingungen als auch des routinierten Einsatzes im Unterrichtsalltag. Eine Studie von Völlinger, Supanc und Brunstein (2018) gibt Hinweise darauf, dass im deutschen Sekundarstufenunterricht KL als ein fester Bestandteil betrachtet werden kann. Nahezu alle befragten Lehrkräfte $(\mathrm{n}=76)$ gaben an, KL regelmäßig durchzuführen, davon fast die Hälfte mindestens zweimal pro Monat. Weitere Befunde der Studie verweisen darauf, dass die empirisch belegten maßgeblichen Merkmale von KL oftmals nicht angemessen berücksichtigt werden.

Bei PISA 2000 wurde die Varianz des Gruppenergebnisses einer kooperativen Problemlöseaufgabe im Wesentlichen durch die kognitiven Problemlösekompetenzen aufgeklärt. Die Autor*innen folgern daraus, dass die "Teamkompetenz“ vor allem mit kognitiven Lernermerkmalen wie etwa kognitiven Fähigkeiten oder Vorwissen zusammenhänge (Kunter, Stanat \& Klieme, 2005). Ein deutlicher Zusammenhang zwischen der Kompetenz kollaborativen Problemlösens und dem Geschlecht wurde in der PISA-Studie 2015 zu Gunsten der Mädchen identifiziert (Zehner, Weis, Vogel, Leutner \& Reiss, 2017).

Berger und Hänze (2004) zeigten in einer Studie, dass sich KL günstig auf die grundlegenden Bedürfnisse der Selbstbestimmungstheorie auswirkte und damit ein höheres $\mathrm{Maß}$ an intrinsischer Motivation und höherwertige Lernstrategien der Lernenden einhergingen. Jurkowski und Hänze (2012) lieferten in verschiedenen Studien Zusammenhänge zwischen Kooperation und Lernerfolg in kooperativen Lernumgebungen. In einer Studie mit Jugendlichen (ebd.) zeigten sie bspw., dass vor allem bei Formen mit stärkerer positiver Interdependenz die kooperativen Fähigkeiten den Lernerfolg positiv beeinflussten.

Darüber hinaus belegen Studien, dass das Trainieren spezifischer Fähigkeiten kooperativer Verhaltensweisen den Lernerfolg steigert. Nach Gillies (2002) zeigten Lernende, die im Hinblick auf lernwirksame kommunikative Aktivitäten ein Training erhalten hatten, mehr Kooperation und häufigeres wechselseitiges Erklären.

Diesen Studien zufolge ist davon auszugehen, dass eine erfolgreiche Kooperation zwischen den Lernenden die Note positiv beeinflussen kann. Tendenziell bewegen sich 
Der Einfluss von Kooperation, Interesse und Motivation auf die Leistungserbringung |

diese Effektstärken in niedrigen, aber oftmals in positiven Bereichen (vgl. Brohm, 2009).

\section{Fragestellungen}

Zu klären gilt es, inwieweit in Prüfungsformen, die explizit die Zusammenarbeit zwischen den Lernenden erfordern, die Kooperation, das Interesse und die Motivation einen Einfluss auf die Prüfungsnote ausüben. Der bisherige Forschungsstand konturiert vor allem die vorangegangenen Schulleistungen, das Geschlecht sowie Interesse und Motivation als bedeutsame Faktoren. Darüber hinaus möchte die vorliegende Studie der Frage nachgehen, ob auch die Kooperation von Schülergruppen zur Vorhersage beiträgt. Daraus ergeben sich die folgenden Forschungsfragen:

1) Inwieweit haben die bisherigen Noten in Deutsch und Mathematik sowie das Geschlecht einen Einfluss auf die Prüfungsnote?

2) Inwiefern klären Motivation und Interesse Varianz in der Bewertung des kooperativen Prüfungsformats auf?

3) Inwiefern beeinflusst die Anzahl der Gruppentreffen die FüK-Note?

4) Inwieweit hängen die Kooperation zwischen den Mitgliedern sowie deren selbst eingeschätzter Nutzen der Kooperation mit der Note zusammen?

Theoriekonform gehen wir davon aus, dass sich die Häufigkeit und die Zusammenarbeit sowie das Interesse am jeweiligen Thema positiv auf die Leistung auswirken. Wir erwarten aufgrund der bisherigen Befunde aus Studien kleine Effektstärken.

\section{Methodenteil}

\subsection{Stichprobe}

Die querschnittliche Befragung der Prüflinge erfolgte an 27 Realschulen und enthält die Angaben von 1.710 Lernenden (45.6\% männlich, 54.4\% weiblich). Das Alter der Probanden betrug $M=16,25$ Jahre ( $S D=.63)$. Bei der Festlegung der Stichprobe wurde auf eine Stratifizierung entlang der Verwaltungsuntereinheiten des Landes Baden-Württemberg zur flächengemäßen Streuung der Schulen in ländlichen und städtischen Regionen, hauptsächlich aber auf differierende Schulgrößen (einzügige bis achtzügige Realschulen) geachtet. Die Datenerhebung erfolgte schriftlich mit einem standardisierten Fragebogen, direkt im Anschluss an die Prüfung der jeweiligen Gruppe und nach Bekanntgabe der Noten der Prüflinge. Die Teilnahme war freiwillig und wurde nur mit elterlichem Einverständnis und der Genehmigung aller Beteiligten durchgeführt. 


\subsection{Instrumente}

Der eingesetzte Fragebogen wurde im Vorfeld im Rahmen einer Pilotstudie $(n=130)$ erprobt. Für die Messung der Konstrukte dienten standardisierte Erhebungsinstrumente, die aufgrund der Feldtestanalysen für die Studie minimal adaptiert wurden. Tabelle 2 gibt einen Überblick über die Skalen und führt zu jeder Skala den Mittelwert, die Standardabweichung und die Reliabilität auf. Bei allen Skalen stellt ein hoher Zahlenwert eine höhere Ausprägung in den gemessenen Merkmalen dar. Die Noten entsprechen der Notenskala des deutschen Schulsystems ( $1=$ sehr gut; $6=$ ungenügend).

Im Folgenden werden die Instrumente kurz beschrieben:

Als Kriterium diente die Zeugnisnote der FüK. Die dazugehörige Häufigkeitsverteilung ist in Abb.1 dargestellt:

Abb. 1: Häufigkeitsverteilung der FüK-Note

\section{Zeugnisnote Klasse 10 in der FüK}

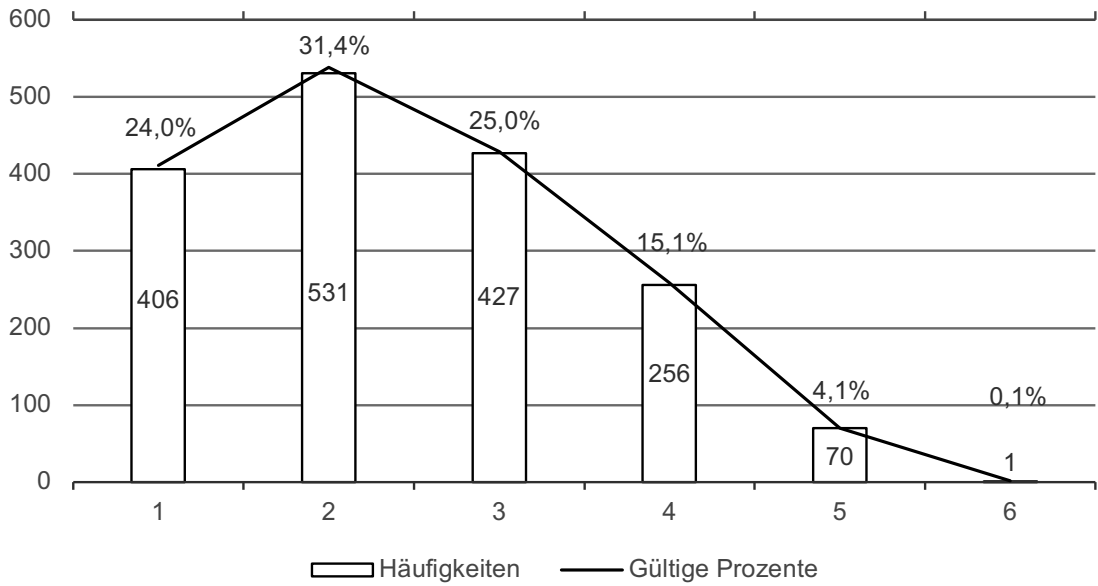

Quelle: eigene Berechnung

Der Mittelwert der FüK-Note betrug $M=2,44$ ( $S D=1,13 ; n=1.691$; fehlend=19). Etwas über die Hälfte aller Prüflinge erreichte eine gute oder sehr gute Note. 
Der Einfluss von Kooperation, Interesse und Motivation auf die Leistungserbringung |

Die Noten in den Fächern Mathematik und Deutsch wurden als Indikator der Schulleistung berücksichtigt; sie gehen als Kontrollvariablen in das Modell ein und beziehen sich auf das Endjahreszeugnis der neunten Klassenstufe. Der Notendurchschnitt in Deutsch betrug $\mathrm{M}=2,82$ ( $\mathrm{SD}=.761 ; \mathrm{n}=1.691$; fehlend=19); in Mathematik lag der Schnitt bei $\mathrm{M}=3,02$ (SD=.888; fehlend=20).

Folgende Instrumente gingen in die Analysen ein:

Zur Erhebung des Interesses am gewählten Thema diente eine aus der PISA-Studie 2000 eingesetzte Skala (Baumert, Gruehn, Heyn, Köller \& Schnabel, 1997; Schiefele, 1991). Die Erhebung der Motivation erfolgte in Anlehnung an die Skala zur aktuellen Motivation aus der Pythagoras-Videostudie (Prenzel, Kirsten, Dengler, Ettle \& Beer, 1996).

Das Format für die Befragung nach der Anzahl der Gruppentreffen ergab sich aus der Feldtestanalyse $(n=130)$. Die erhobenen Antwortmöglichkeiten sowie eine Häufigkeitsverteilung der Schülerangaben zeigt Tabelle 1.

Tab. 1: Häufigkeiten der Gruppentreffen

\begin{tabular}{|l|c|c|c|c|}
\hline Anzahl der Gruppentreffen & keine & 1 bis 3 & 4 bis 6 & öfter als 6 \\
\hline Häufigkeiten & 81 & 482 & 598 & 536 \\
\hline Gültige Prozente & 4,8 & 28,4 & 35,2 & 31,6 \\
\hline
\end{tabular}

Quelle: eigene Berechnung

Zur Erhebung der Kooperation wurde die Skala von Helmke et al. (2011) aus EMU (Evidenzbasierte Methoden der Unterrichtsdiagnostik und -entwicklung) herangezogen.

Die Erfassung des wahrgenommenen Nutzens der Gruppenarbeit erfolgte mit der Skala zur „Partner- und Gruppenarbeit: Wahrgenommener Nutzen“ von Stebler und Reusser (1995) aus der TIMSS +-Studie im Nationalfonds-Projekt Schule, Leistung und Persönlichkeit (1995). 
Tab. 2: $\quad$ Übersicht der Skalen

\begin{tabular}{|c|c|c|c|c|}
\hline Skala & $M$ & $S D$ & $\begin{array}{c}\text { Cron- } \\
\text { bachs } \alpha\end{array}$ & Einzelne Items \\
\hline Interesse & 3.03 & 0,64 & .81 & $\begin{array}{l}\text { Ich fand unser Gruppenthema interessant. (1) } \\
\text { Ich fand mein Schwerpunktthema interessant. (2) } \\
\text { Es war mir wichtig, mehr über mein Schwerpunktthema zu } \\
\text { erfahren. (3) } \\
\text { Die Beschäftigung mit meinem Schwerpunktthema hat mir } \\
\text { Spaß gemacht. (4) } \\
\text { Im Vergleich zu anderen Dingen, mit denen ich mich beschäfti- } \\
\text { ge, hatte mein Thema nur wenig Bedeutung für mich. (5) }\end{array}$ \\
\hline Motivation & 2.88 & 0,69 & .82 & $\begin{array}{l}\text { Während ich mich mit meinem Schwerpunktthema beschäftigt } \\
\text { habe, ... } \\
\ldots \text { hat mir die Arbeit Spaß gemacht. (1) } \\
\ldots \text { wollte ich den Stoff wirklich verstehen. (2) } \\
\ldots \text { hat mich die Sache so fasziniert, dass ich mich voll einsetz- } \\
\text { te. (3) } \\
\ldots \text { hatte ich keine Lust, mich mit den Inhalten zu beschäfti- } \\
\text { gen. (4) } \\
\ldots \text { war ich mit meinen Gedanken woanders. }\end{array}$ \\
\hline $\begin{array}{l}\text { Koope- } \\
\text { ration }\end{array}$ & 3.37 & .60 & .83 & $\begin{array}{l}\text { In meiner Gruppe haben wir uns gegenseitig unterstützt. (1) } \\
\text { Die Verteilung der Aufgaben innerhalb der Gruppe war fair. (2) } \\
\text { Alle Meinungen wurden ernst genommen. (3) } \\
\text { Wenn jemand gesprochen hat, haben die anderen zugehört. (4) } \\
\text { Keiner wurde links liegen gelassen. (5) } \\
\text { Probleme wurden offen angesprochen. (6) }\end{array}$ \\
\hline $\begin{array}{c}\text { Nutzen der } \\
\text { Gruppen- } \\
\text { arbeit }\end{array}$ & 2.86 & .68 & .85 & $\begin{array}{l}\text { Durch unsere Gruppenarbeit ... } \\
\ldots \text { habe ich unser Thema besser verstanden. (1) } \\
\ldots \text { habe ich Neues zu unserem Thema dazugelernt. (2) } \\
\ldots \text { habe ich neue Ideen zum Weiterdenken bekommen. (3) } \\
\ldots \text { konnten wir Dinge lösen, die für mich alleine zu schwierig } \\
\text { waren. (4) } \\
\ldots \text { haben mir die Erklärungen der anderen weitergeholfen. (5) } \\
\ldots \text { profitierte ich von der Art und Weise, wie die anderen an die } \\
\text { Dinge herangingen. (6) }\end{array}$ \\
\hline
\end{tabular}

Anm.: Antwortformat: 1 = stimme nicht $\mathrm{zu}, 2=$ stimme eher nicht $\mathrm{zu}, 3=$ stimme eher $\mathrm{zu}$, $4=$ stimme voll $\mathrm{zu}$

Quelle: eigene Berechnung

\subsection{Statistische Analysen}

Die Gruppenzugehörigkeit in der Stichprobe ist ein möglicher Kontextfaktor, welcher sich dadurch auszeichnen könnte, dass sich Lernende innerhalb der Gruppe ähnlicher sind als Personen aus unterschiedlichen Gruppen. Dies könnte dazu führen, dass Signifikanztests zu liberal beurteilt werden (Raudenbush \& Bryk, 2002). Bei den statistischen Analysen wurde deswegen die Gruppenzugehörigkeit über eine Korrektur der Standardfehler berücksichtigt (vgl. Muthén \& Muthén, 1998-2012). Zudem wur- 
Der Einfluss von Kooperation, Interesse und Motivation auf die Leistungserbringung |

de ein Verfahren gewählt (Full Informationen Maximum Likelihood-Verfahren; vgl. Lüdtke, Robitzsch, Trautwein \& Köller, 2007), das fehlende Werte berücksichtigt.

Zur Prüfung, ob die Prädiktoren einen Einfluss auf die Note haben, wurde eine hierarchische lineare Regressionsanalyse berechnet mit Dummy-kodierten Variablen für die Anzahl der Gruppentreffen (vgl. Tab. 1). Die Analyse erfolgte in vier Schritten. Zunächst wurden die Kontrollvariablen (Geschlecht; Mathematik- und Deutschnote) zur Vorhersage der Leistung herangezogen. Im zweiten Schritt erfolgte die Aufnahme der Interessens- und Motivationsskalen. Im dritten Schritt ging die Anzahl der Gruppentreffen in das Modell ein. In Schritt vier wurde als Prädiktor die Skala zur Kooperation und zum Nutzen der Gruppenarbeit aufgenommen.

Berichtet werden die Korrelationen $(r)$ der Prädiktoren mit dem Kriterium, die standardisierten Regressionsgewichte ( $\beta$-Gewicht), der multiple Determinationskoeffizient $R^{2}$ und die Veränderung des multiplen Determinationskoeffizienten $\Delta R^{2}$.

\section{Ergebnisse}

Tabelle 3 veranschaulicht die Ergebnisse der Regressionsmodelle auf die Note.

Im ersten Modell bestätigte sich der positive Zusammenhang zwischen den bisherigen Schulnoten und der FüK-Note. Die Prädiktoren erzielten eine Varianzaufklärung von 17,4 Prozent $(p<.001)$. Entgegen unseren Erwartungen ließ sich für das Geschlecht kein signifikanter Einfluss nachweisen.

Der Einbezug des Interesses und der Motivation im zweiten Schritt klärt zusätzlich zum ersten Modell weitere 11,2 Prozent Varianz auf $(p<.001)$. Das Interesse erwies sich mit $\beta=-0.25(p<.001)$ als Prädiktor mit der stärksten Vorhersagekraft. Das negative Vorzeichen erklärt sich durch die Kodierung der Noten $(1=\operatorname{sehr}$ gut; $6=$ ungenügend). Der Einfluss der bisherigen Noten bleibt durch die Hinzunahme des Prädiktoren-Sets hoch signifikant, verliert aber etwas an Einflussgröße. Insgesamt werden durch die ersten beiden Modelle 29 Prozent der Kriteriumsvarianz aufgeklärt $(p<.001)$.

Inwieweit in diesem kooperativen Prüfungsformat der Kooperation ein Einfluss auf den Prüfungserfolg zukommt, wurde in den Modellen drei und vier geprüft.

Modell drei klärt auf, inwieweit die Anzahl der Gruppentreffen mit der FüK-Note zusammenhängt. Unter Hinzunahme dieser Variable zeigt sich ein signifikanter Einfluss auf die Abschlussnote. Es bestätigten sich unsere Erwartungen, dass Lernende einer Gruppe, die sich öfter getroffen hatte, eine bessere Note in der Prüfung erreichten $\left(R^{2}=.30, p<.001\right)$. 
Tab. 3: $\quad$ Ergebnisse der Regressionsanalyse zur Vorhersage der FüK-Note

\begin{tabular}{|c|c|c|c|c|c|c|c|c|c|}
\hline \multirow[b]{2}{*}{ Prädiktoren } & \multirow[b]{2}{*}{$r$} & \multicolumn{2}{|c|}{ Modell 1} & \multicolumn{2}{|c|}{ Modell 2} & \multicolumn{2}{|c|}{ Modell 3} & \multicolumn{2}{|c|}{ Modell 4} \\
\hline & & $\beta(p)$ & SE $(\beta)$ & $\beta(\mathbf{p})$ & SE $(\beta)$ & $\beta(p)$ & SE ( $\beta)$ & $\beta(p)$ & SE $(\beta)$ \\
\hline Deutschnote ${ }^{a}$ & 0.348 & $0.248^{\star \star *}$ & 0.029 & $0.215^{\star \star \star}$ & 0.027 & $0.208^{* * *}$ & 0.027 & $0.217^{\star \star *}$ & 0.027 \\
\hline Mathenote ${ }^{b}$ & 0.326 & $0.245^{\star * *}$ & 0.027 & $0.223^{\star * *}$ & 0.025 & $0.213^{* * *}$ & 0.025 & $0.213^{* * *}$ & 0.025 \\
\hline Geschlecht ${ }^{c}$ & -0.136 & -0.057 & 0.033 & -0.025 & 0.030 & -0.009 & 0.030 & -0.001 & 0.030 \\
\hline Interesse & -0.384 & & & $-0.245^{\star \star *}$ & 0.034 & $-0.250^{\star \star *}$ & 0.034 & $-0.237^{\star \star \star}$ & 0.034 \\
\hline Motivation & -0.347 & & & $-0.119^{\star \star \star}$ & 0.033 & $-0.098^{* *}$ & 0.033 & -0.061 & 0.033 \\
\hline 0-3 Treffen & 0.197 & & & & & $0.112^{\star * *}$ & 0.031 & $0.084^{\star *}$ & 0.033 \\
\hline 4-6 Treffen & 0.027 & & & & & $0.097^{\star *}$ & 0.031 & $0.098^{\star *}$ & 0.031 \\
\hline Kooperation ${ }^{d}$ & -0.213 & & & & & & & $-0.056^{*}$ & 0.027 \\
\hline Nutzen Gruppe ${ }^{\ominus}$ & -0.252 & & & & & & & $-0.082^{\star \star}$ & 0.029 \\
\hline \multicolumn{2}{|l|}{$R^{2}$} & \multicolumn{2}{|c|}{$0.174^{\star \star \star}$} & \multicolumn{2}{|c|}{$0.286^{\star \star \star}$} & \multicolumn{2}{|c|}{$0.297^{* * *}$} & \multicolumn{2}{|c|}{$0.309^{* * *}$} \\
\hline \multicolumn{2}{|l|}{$\Delta R^{2}$} & & & \multicolumn{2}{|c|}{$0.112^{\star \star \star}$} & \multicolumn{2}{|c|}{$0.011^{* * *}$} & \multicolumn{2}{|c|}{$0.012^{\star * *}$} \\
\hline
\end{tabular}

Anm.: $\quad r=$ Pearson Korrelation; $\beta=$ Standardisierter Regressionskoeffizient;

$S E(\beta)=$ Standardfehler von $\beta ; R^{2}=$ Multipler Determinationskoeffizient; $\Delta R^{2}=$ Änderung

des Multiplen Determinationskoeffizienten; ${ }^{*} p<.05,{ }^{* *} p<.01,{ }^{* * *} p<.001$

${ }^{a}$ Deutschnote für die Deutschnote im Zeugnis Ende Klasse 9

${ }^{\mathrm{b}}$ Mathenote für die Mathematiknote im Zeugnis Ende Klasse 9

${ }^{\mathrm{c}}$ Geschlecht: männlich $=0$; weiblich $=1$

${ }^{\mathrm{d}}$ Kooperation für Kooperation in der Gruppe

${ }^{\mathrm{e}}$ Nutzen Gruppe für den selbsteingeschätzten Nutzen der Gruppenarbeit

Die Referenzkategorie für die Dummy-kodierten Variablen der Gruppentreffen wurde aus

Antwortmöglichkeit 4 (öfter als 6 Mal getroffen) gebildet.

Quelle: eigene Berechnung

Um zu prüfen, inwieweit es sich bei diesem Einfluss möglicherweise um einen Effekt der gelungenen Kooperation zwischen den Mitgliedern sowie des subjektiv empfundenen Nutzens der Gruppenarbeit handelt, wurden in einem vierten Schritt die Prädiktoren Kooperation und Nutzen der Gruppenarbeit aufgenommen. Beide Prädiktoren liefern einen substanziellen Beitrag zur Varianzaufklärung $\left(R^{2}=.31\right.$; $p<.001$ ), wenngleich dieser jedoch in seiner Höhe klein ausfällt. Der Einfluss der Motivation verliert durch den Einbezug der zwei Prädiktoren das Signifikanzniveau. Weiterhin erweist sich das Interesse mit $\beta=-0.24(p<.001)$ als stärkster Prädiktor zur Vorhersage der Prüfungsnote.

Zusammenfassend zeigt sich, dass der Anteil der aufgeklärten Varianz zur Vorhersage der Note in der FüK für alle Merkmalsgruppen insgesamt $R^{2}=.31(p<.001)$ beträgt. Der Varianzzuwachs zwischen den Modellen ist dabei zwar statistisch signifikant, aber relativ betrachtet mit jeweils gut einem Prozent eher gering. Relevant zur Vorhersage 
Der Einfluss von Kooperation, Interesse und Motivation auf die Leistungserbringung |

der Prüfungsnote sind - nach Kontrolle der Noten in Deutsch und Mathematik insbesondere das themenbezogene Interesse, die Häufigkeit der Gruppentreffen, die Kooperation und der subjektiv empfundene Nutzen der Gruppentreffen.

\section{Zusammenfassung und Diskussion}

In dieser Studie wurde untersucht, inwieweit Intensität und subjektives Empfinden kooperativer Handlungen in kooperativen Prüfungsformaten mit der erreichten Note zusammenhängen. Die Befunde der Schülerbefragung $(n=1.670)$ beziehen sich auf die Merkmale der in Baden-Württemberg implementierten FüK, einer kooperativen Abschlussprüfung. Die Auswertung der Daten erfolgte über eine multiple Regressionsanalyse.

Als ein wesentlicher Befund lässt sich die Frage, ob die Kooperation mit der erreichten Note zusammenhängt, positiv beantworten. Sowohl die Anzahl der Gruppentreffen als auch die empfundene Qualität der Kooperation scheinen sich auf die Note auszuwirken: Gruppen, die sich öfter getroffen hatten und die Zusammenarbeit positiv beurteilten, erreichten eine bessere Note. Theoriekonform zu bisherigen Befunden hing die Note auch vom Zusammenhalt und von der wechselseitigen Unterstützung der Gruppenmitglieder ab. Lernende, die angaben, dass in ihren Sitzungen interdependente Bedingungen herrschten und ein reger, positiver Austausch zwischen ihren Gruppenmitgliedern stattfand, erzielten bessere Noten.

Die stärkste Vorhersagekraft zeigte sich aber durch das Interesse der Lernenden. Möglicherweise kommen hierbei die in der Selbstbestimmungstheorie vorausgesetzten Bedürfnisse zum Tragen. Die vielfältigen Wahl- und Handlungsmöglichkeiten dieser Arbeitsweise bieten den Lernenden einen großen Spielraum zum eigenständigen Entscheiden und interessengeleiteten Handeln.

Die verwendeten Prädiktoren konnten insgesamt die Varianz der Prüfungsnote zu 31 Prozent aufklären. Ein größerer Anteil der Variabilität bleibt damit zunächst ungeklärt. Betrachtet man die Tatsache, dass in der FüK nur ganze Noten vergeben werden, so ist festzuhalten, dass durch diese grobe Maßeinheit die Varianz des erzielten Prüfungserfolgs eingeschränkt wird. Des Weiteren konnten in der Studie weder die von den Prüfungskommissionen definierten curricularen Kriterien der Notenvergabe noch die prozentuale Aufteilung der einzelnen Kompetenzbereiche in der Gesamtnote beachtet werden. In der Literatur wird auf erhebliche Schwierigkeiten in der Bewertung kooperativer Prüfungen hingewiesen (vgl. Döring, Strobel-Eisele, Wacker, Heizmann \& Kramer, 2016; Maag Merki, 2004). Das Problem liegt u.a. darin, dass die Zertifizierung häufig über die Form der Präsentationsprüfung vorgenommen wird, in der jeder Prüfling einen Teil des Themas übernimmt. Die Studie von Döring et al. (2016) zeigt, dass dabei der Fachkompetenz das größte Gewicht zukommt. Die Frage 
bleibt offen, inwieweit sich auf diese Weise Kooperationskompetenzen bewerten lassen. Die in der Studie letztlich aufgefundenen niedrigen Zusammenhänge können als ein Hinweis auf diesen Sachverhalt interpretiert werden. Inwieweit die Notenvergabe die Prädiktoren beeinflusst, stellt ein Forschungsdesiderat für weitere Studien dar.

Bei der Interpretation der Befunde sind weitere Einschränkungen zu berücksichtigen. Erstens ist eine Generalisierbarkeit auf kooperative Prüfungsformate in anderen Bundesländern oder Schulformen nicht möglich, denn sowohl deren Konzeptionen als auch die zu Grunde liegenden Bildungspläne variieren. Zweitens ist anzumerken, dass sich die Erhebung ausschließlich auf Selbstauskünfte der Jugendlichen stützte. Vor allem der hoch sensible Erhebungszeitpunkt, der direkt nach der Prüfung und unmittelbar nach der Notenbekanntgabe stattfand, kann mit einem Einfluss auf die dargelegten Selbsteinschätzungen der Lernenden verbunden sein. Dieser könnte bspw. zu künstlichen Effekten führen. Eine Befragung vor der FüK war jedoch aus rechtlichen Gründen, insbesondere im Hinblick auf eine dadurch befürchtete Beeinflussung der Beurteilungssituation, unmöglich und hätte die Studie insgesamt verhindert. Zudem galt es, den Fragebogen kurz zu gestalten, um unter diesen Bedingungen die Teilnahmemotivation aufrechtzuerhalten. Damit könnten Informationsverluste einhergegangen sein. Bei der Interpretation der Ergebnisse ist dieser Sachverhalt besonders zu beachten.

Dennoch vermag die Studie wichtige Hinweise darauf zu geben, dass neben den bisherigen Noten der Lernenden sowohl die Kooperation als auch das Interesse im Zusammenhang mit der erreichten Prüfungsnote stehen. Um diese Aspekte genauer betrachten zu können, sollten zusätzliche längsschnittlich angelegte Studien durchgeführt werden und somit die aufgezeigten Einflussgrößen repliziert bzw. weitere identifiziert werden. Wenn es gelänge, bestimmte Faktoren zu bestimmen, die für effektive Gruppenarbeit im Schulkontext maßgeblich sind, wären dies Ansatzpunkte für weitere Überlegungen zur Erfassung, aber auch zur gezielten Förderung von Teamkompetenzen. Dies würden wichtige Grundlagen für die Konzeption gezielter Interventionsstudien zur Förderung von Kooperationskompetenzen darstellen. Die Ergebnisse aus aktuellen Interventionsstudien, die das Ziel verfolgten, sozialbezogene Kompetenzen zu steigern, verweisen auf erhebliche Schwierigkeiten bezüglich der Messbarkeit dieser Kompetenzbereiche (z. B. Brohm, 2009; Döring, Strobel-Eisele, Wacker, Heizmann \& Kramer, 2018). In dieser Hinsicht wären längerfristig angelegte Interventionsstudien ein dringendes Forschungsdesiderat.

Auch wenn die vorliegenden Ergebnisse nicht ohne weiteres übertragbar sind, tritt mit den Befunden insbesondere die schulische Förderung der Kooperationskompetenz von Lernenden als Aufgabe in den Mittelpunkt. Es ist davon auszugehen, dass eine effektive Kooperation eine sorgfältige Implementation der Methode sowie deren Erweiterung und Vertiefung durch gezielte Trainings über die Schulzeit eines Lernenden hinweg erfordert. Im Unterricht fest verankerte und über die 
Der Einfluss von Kooperation, Interesse und Motivation auf die Leistungserbringung |

Jahrgangsstufen im Sinne eines Spiralcurriculums hinweg konzipierte Maßnahmen zur Etablierung kooperativer Verhaltensweisen scheinen eine wichtige Voraussetzung zur Entwicklung von Teamkompetenzen und für den Erfolg in diesen Lernarrangements zu sein.

\section{Literatur und Internetquellen}

Baumert, J., Gruehn, S., Heyn, S., Köller, O., \& Schnabel, K.-U. (1997). Bildungsverläufe und psychosoziale Entwicklung im Jugendalter (BIJU). Dokumentation, Bd. 1: Skalen Längsschnitt 1, Welle 1-4. Berlin: Max-Planck-Institut für Bildungsforschung.

Berger, R., \& Hänze, M. (2004). Das Gruppenpuzzle im Physikunterricht der Sekundarstufe II - Einfluss auf Motivation, Lernen und Leistung. Zeitschrift für Didaktik der Naturwissenschaft, 10, 205-219.

Borsch, F. (2015). Kooperatives Lernen: Theorie, Anwendung, Wirksamkeit (2., überarb. und erweiterte Aufl.). Stuttgart: Kohlhammer.

Brohm, M. (2009). Sozialkompetenz und Schule. Theoretische Grundlagen und empirische Befunde zu Gelingensbedingungen sozialbezogener Interventionen. Weinheim \& München: Juventa.

Brüning, L., \& Saum, T. (2009a). Erfolgreich unterrichten durch Kooperatives Lernen, Bd. 1: Strategien zur Schüleraktivierung (5., überarb. Aufl). Essen: Neue Deutsche Schule.

Brüning, L., \& Saum, T. (2009b). Erfolgreich unterrichten durch Kooperatives Lernen, Bd. 2: Neue Strategien zur Schüleraktivierung: Individualisierung, Leistungsbeurteilung, Schulentwicklung. Essen: Neue Deutsche Schule.

COOL - „Co-Operative Open Learning“. Zugriff am 03.11.2018. Verfügbar unter: https:// www.cooltrainers.at $/$ index.php?id $=518 \& \mathrm{~L}=-1 \% 252527 \% 252522$.

Deci, E. L., \& Ryan, R. M. (2002). Handbook of Self-Determination Research. Rochester: University of Rochester.

Döring, M., Strobel-Eisele, G., Wacker, A., Heizmann, E., \& Kramer, J. (2016). Kompetenzorientiert Prüfen in der Sekundarstufe. Eine empirische Studie zur Fächerübergreifenden Kompetenzprüfung an Realschulen in Baden-Württemberg. In S. Hadeler, K. Moegling \& G. Hund-Göschel (Hrsg.), Was sind gute Schulen? Teil 3: Forschungsergebnisse (S. 29-43). Immenhausen: Prolog.

Döring, M., Strobel-Eisele, G., Wacker, A., Heizmann, E., \& Kramer, J. (2018). Soziale Kompetenzen als Bestandteil von Abschlussprüfungen in der Sekundarstufe - Empirische Befunde. Lernen und Lernstörungen, 7, 7-20. Online publiziert am 06.10.2017. https:// doi.org/10.1024/2235-0977/a000196

Dubs, R. (2009). Lehrerverhalten. Ein Beitrag zur Interaktion von Lehrenden und Lernenden im Unterricht. Stuttgart: Franz Steiner.

Gillies, R. M. (2002). The Residual Effects of Cooperative-Learning Experiences: A Two-Year Follow-up. Journal of Educational Research, 96 (1), 15-20. https://doi. org/10.1080/00220670209598787

Green, N., \& Green, K. (2005). Kooperatives Lernen im Klassenraum und im Kollegium. Seelze: Kallmeyer.

Hamm, J. V., \& Faircloth, B. S. (2005). The Role of Friendship in Adolescents' Sense of School Belonging. New Directions for Child and Adolescent Development, 107, 61-78. https://doi.org/10.1002/cd.121

Hattie, J. (2013). Lernen sichtbar machen. Baltmannsweiler: Schneider Verlag Hohengehren.

Helmke, A. (2015). Unterrichtsqualität und Lehrerprofessionalität. Diagnose, Evaluation und Verbesserung des Unterrichts (6. Aufl.). Seelze: Klett-Kallmeyer. 
Helmke, A., Helmke, T., Lenske, G., Pham, G., Praetorius, A.-K., Schrader, F.-W., et al. (2011). EMU - Unterrichtsdiagnostik. Version 3.2 Kultusministerkonferenz. Landau: Universität Koblenz-Landau, Campus Landau.

Huber, A. (2007). Wechselseitiges Lehren und Lernen (WELL) als spezielle Form des Kooperativen Lernens. Berlin: Logos.

Johnson, D. W., \& Johnson, R. T. (2006). Joining Together: Group Theory and Group Skills (9. Aufl.). Boston, MA: Allyn \& Bacon.

Jurkowski, S., \& Hänze, M. (2012). Kooperatives Lernen aus dem Blickwinkel sozialer Kompetenzen. Unterrichtswissenschaft, 40 (3), 259-276.

Kanning, U. P. (2009). Diagnostik sozialer Kompetenzen (2., aktualisierte Aufl.). Göttingen: Hogrefe.

Kohler, B., \& Wacker, A. (2013). Das Angebot-Nutzungs-Modell. Überlegungen zu Chancen und Grenzen des derzeit prominentesten Wirkmodells der Schul- und Unterrichtsforschung. DDS - Die Deutsche Schule, 105 (3), 242-258.

Konrad, K., \& Traub, S. (2010). Kooperatives Lernen. Baltmannsweiler: Schneider Verlag Hohengehren.

Krapp, A. (2005). Basic Needs and the Development of Interest and Intrinsic Motivational Orientations. Learning and Instruction, 15 (5), 381-395. https://doi.org/10.1016/j.learn instruc.2005.07.007

Krapp, A. (2008). Motivation ist das A und O. Über eine grundlegende Gelingensbedingung kooperativen Lernens. Friedrich Jahresheft, 26, 79-81.

Kunter, M., Stanat, P., \& Klieme, E. (2005). Die Rolle von individuellen Eingangsvoraussetzungen und Gruppenmerkmalen beim kooperativen Lösen eines Problems. In E. Klieme (Hrsg.), Diagnostische Ansätze, theoretische Grundlagen und empirische Befunde der deutschen PISA-2000-Studie (S. 99-115). Wiesbaden: VS. https://doi. org/10.1007/978-3-322-85144-4_8

Lüdtke, O., Robitzsch, A., Trautwein, U., \& Köller, O. (2007). Umgang mit fehlenden Werten in der psychologischen Forschung. Psychologische Rundschau, 58 (2), 103-117. https:// doi.org/10.1026/0033-3042.58.2.103

Maag Merki, K. (2004). Überfachliche Kompetenzen als Ziele beruflicher Bildung im betrieblichen Alltag. Zeitschrift für Pädagogik, 50 (2), 202-222.

Marsh, H. W., Trautwein, U., Lüdtke, O., Köller, O., \& Baumert, J. (2005). Academic SelfConcept, Interest, Grades, and Standardized Test Scores: Reciprocal Effects Models of Causal Ordering. Child Development, 76 (2), 397-416. https://doi.org/10.1111/j.14678624.2005.00853.x

MKJS BW (Ministerium für Kultus, Jugend und Sport Baden-Württemberg) (2006). Realschule. Neue Abschlussprüfung. Handreichung zur neuen Realschulabschlussprüfung. Stuttgart: Ministerium.

Müller, K., \& Bungard, W. (2013). Team- und Kooperationsfähigkeit. In W. Sarges (Hrsg.), Management-Diagnostik (S. 385-395). Göttingen: Hogrefe.

Muthén, L. K., \& Muthén, B. O. (1998-2012). Mplus - Statistical Analysis with Latent Variables. Los Angeles, CA: Muthén \& Muthén.

Prenzel, M., Kirsten, A., Dengler, P., Ettle, R., \& Beer, T. (1996). Selbstbestimmt motiviertes und interessiertes Lernen in der kaufmännischen Erstausbildung. In K. Beck \& H. Heid (Hrsg.), Lehr-Lern-Prozesse in der kaufmännischen Erstausbildung: Wissenserwerb, Motivierungsgeschehen und Handlungskompetenzen (Zeitschrift für Berufs- und Wirtschaftspädagogik, 13. Beiheft) (S. 108-127). Stuttgart: Steiner.

Raudenbush, S. W., \& Bryk, A. S. (2002). Hierarchical Linear Models: Applications and Data Analysis Methods (2. Aufl.). Thousand Oaks, CA: Sage. 
Der Einfluss von Kooperation, Interesse und Motivation auf die Leistungserbringung |

Reinmann, G., \& Mandl, H. (2006). Unterrichten und Lernumgebungen gestalten. In A. Krapp \& B. Weidenmann (Hrsg.), Pädagogische Psychologie. Ein Lehrbuch (5. Aufl.) (S. 613-656). Weinheim \& Basel: Beltz.

Schiefele, U. (1991). Interest, Learning, and Motivation. Educational Psychologist, 26 (3-4), 299-323. https://doi.org/10.1080/00461520.1991.9653136

SINUS-Transfer - „Steigerung der Effizienz des mathematisch-naturwissenschaftlichen Unterrichts“. Modul 8: Kooperatives Lernen. Zugriff am 03.11.2018. Verfügbar unter: http://www.sinus-transfer.de/module/modul_8kooperatives_lernen.html.

Slavin, R. E. (1993). Kooperatives Lernen und Leistung: Eine empirisch fundierte Theorie. In G. L. Huber (Hrsg.), Neue Perspektiven der Kooperation (S. 151-170). Baltmannsweiler: Schneider Verlag Hohengehren.

Stebler, R., \& Reusser, K. (1995). Skalendokumentation der Schweizerischen Zusatzerhebungen $z u$ TIMSS. Zürich: Universität Zürich, Pädagogisches Institut.

Völlinger, V., Supanc, M., \& Brunstein, J. (2018). Kooperatives Lernen in der Sekundarstufe. Häufigkeit, Qualität und Bedingungen des Einsatzes aus der Perspektive der Lehrkraft. Zeitschrift für Erziehungswissenschaft, 21 (1), 159-176. https://doi.org/10.1007/s11618017-0764-0

Vygotsky, L. S. (1978). Mind in Society: The Development of Higher Psychological Processes. Cambridge, MA: Harvard University Press.

Zehner, F., Weis, M., Vogel, F., Leutner, D., \& Reiss, K. (2017). Kollaboratives Problemlösen in PISA 2015: Befundhöhepunkte rund um Deutschland. München: Technische Universität München. Zugriff am 12.04.2018. Verfügbar unter: https://www.pisa.tum.de/fileadmin/ w00bgi/www/Berichtsbaende_und_Zusammenfassungungen/Kurzbericht_CPS_2015_ ZIB_final.pdf.

Elke Heizmann, geb. 1976, Mitglied des Kooperativen Promotionskollegs „Effektive Lehr-Lernarrangements“ der Pädagogischen Hochschule Ludwigsburg und der Universität Tübingen.

E-Mail: heizmann@ph-ludwigsburg.de

Gabriele Strobel-Eisele, Prof. Dr. habil., geb. 1953, Professorin für Erziehungswissenschaft mit dem Schwerpunkt Schulpädagogik an der Pädagogischen Hochschule Ludwigsburg.

E-Mail: strobeleisele@ph-ludwigsburg.de

Melanie Döring, Dipl.-Päd., geb. 1984, Mitglied des Kooperativen Promotionskollegs „Effektive Lehr-Lernarrangements“ der Pädagogischen Hochschule Ludwigsburg und der Universität Tübingen.

E-Mail: ga_doering@ph-ludwigsburg.de

Korrespondenzadresse: Pädagogische Hochschule Ludwigsburg, Reuteallee 46, 71634 Ludwigsburg

Albrecht Wacker, Prof. Dr., geb. 1969, Professor für Erziehungswissenschaft mit dem Schwerpunkt Schulpädagogik - Pädagogik der Sekundarstufe I an der Pädagogischen Hochschule Heidelberg.

E-Mail: wacker@ph-heidelberg.de 
| E. Heizmann, A. Wacker, G. Strobel-Eisele, M. Döring, J. Kramer \& C. Rietz

Christian Rietz, Prof. Dr. phil., Dipl.-Psych., geb.1964, Professur für Mixed-MethodsForschung, Institut für Erziehungswissenschaft an der Pädagogischen Hochschule Heidelberg.

E-Mail: christian.rietz@ph-heidelberg.de

Korrespondenzadresse: Pädagogische Hochschule Heidelberg, Keplerstraße 87, 69120 Heidelberg

Jochen Kramer, Dr. phil., Dipl.-Psych., geb. 1973, Projektleiter, Türkische Gemeinde in Baden-Württemberg.

E-Mail: jochen.kramer@tgbw.de

Korrespondenzadresse: Türkische Gemeinde in Baden-Württemberg (tgbw), Reinsburgstraße 82, 70178 Stuttgart 